\begin{tabular}{|c|c|}
\hline Title & Terahertz Emission of Radiation from InGaP/nGaA s/GaA s Grating-Bicoupled Plasmon-Resonant Photomixer \\
\hline Author(s) & Otsuji, Taiichi; Hanabe, Mitsuhiro; Meziani, Y ahya M.; Sano, Eiichi \\
\hline Citation & $\begin{array}{l}\text { Device Research Conference, } 2006 \text { 64th, 193-194 } \\
\text { https://doi.org/10.1109/DRC.2006.305052 }\end{array}$ \\
\hline Issue Date & $2006-06$ \\
\hline Doc URL & http://hdl.handle.net/2115/28782 \\
\hline Rights & $\begin{array}{l}\text { (C2006 IEEE. Personal use of this material is permitted. However, permission to reprint/republish this material for } \\
\text { advertising or promotional purposes or for creating new collective works for resale or redistribution to servers or lists, } \\
\text { or to reuse any copyrighted component of this work in other works must be obtained from the IEEE. IEEE, Device } \\
\text { Research Conference, } 2006 \text { 64th, 2006, 193-194. }\end{array}$ \\
\hline Type & proceedings \\
\hline Note & 64th Device Research Conference, The PennsyIvania State University, University Park, Pennsylvania, June 26- 28, 2006 \\
\hline File Information & DRC2006.pdf \\
\hline
\end{tabular}

Instructions for use 
June 26 - 28, 2006

The Pennsylvania State University University Park, Pennsylvania
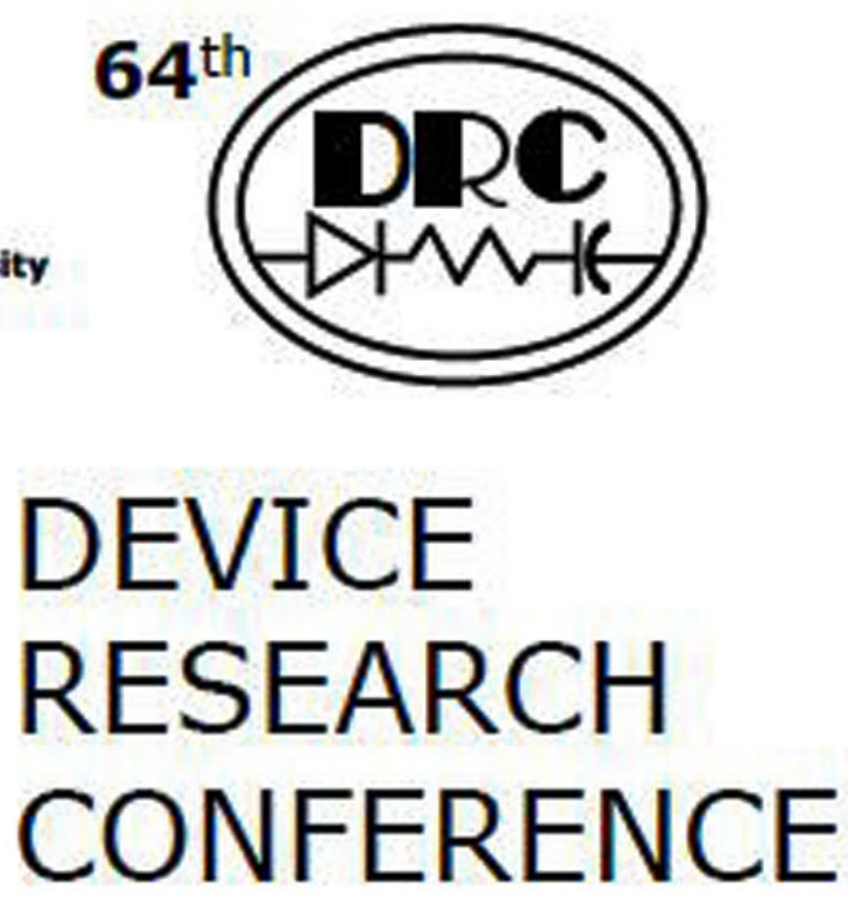

Q IEEE

RESEARCH

CONFERENCE

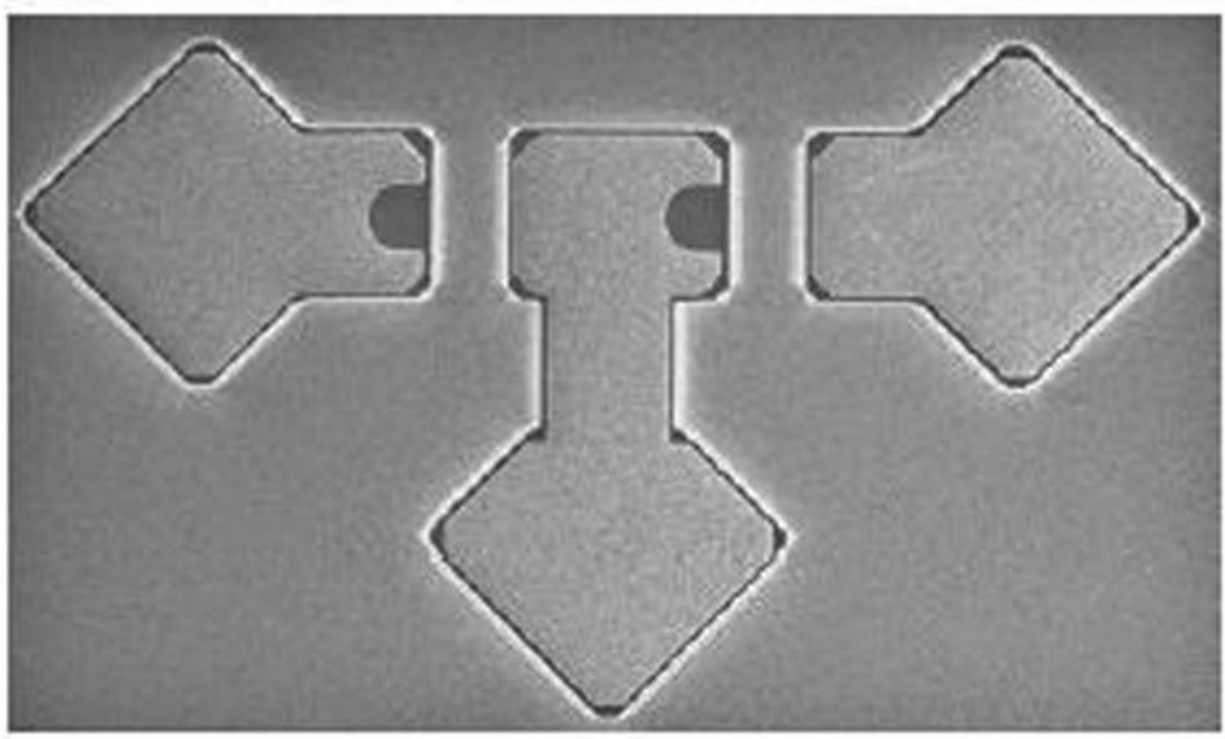

Conference Digest 


\title{
Terahertz Emission of Radiation from InGaP/InGaAs/GaAs Grating-Bicoupled Plasmon-Resonant Photomixer
}

\author{
Taiichi Otsuji ${ }^{1}$, Mitsuhiro Hanabe ${ }^{1,}$ Yahya M. Meziani ${ }^{1}$, and Eiichi Sano ${ }^{2}$ \\ ${ }^{1}$ Research Institute of Electrical Communication, Tohoku University, Sendai, 980-8577, Japan \\ Email: otsuji@riec.tohoku.ac.jp; hanabe@riec.tohoku.ac.jp; yahya@riec.tohoku.ac.jp \\ ${ }^{2}$ Research Center for Integrated Quantum Electronics, Hokkaido University, Sapporo, 060-8628, Japan \\ Email: esano@rciqe.hokudai.ac.jp
}

Two dimensional (2D) plasma-wave instabilities in submicron transistors have attracted much attention due to their nature of promoting emission of electro-magnetic radiation in the terahertz range [1-5]. We have designed and fabricated our original terahertz plasmon-resonant photomixer incorporating doubly interdigitated grating gates and a vertical cavity into a HEMT structure [4], succeeding in the first observation of stimulated emission of terahertz radiation at room temperature.

The device was fabricated with InGaP/InGaAs/GaAs material systems as shown in Fig. 1. The 2D plasmon layer is formed with a quantum well in the InGaAs channel layer. The grating gate was formed with 65-nm thick Ti/Au/Ti by a standard lift-off process. To cover operating frequencies from 1 to $10 \mathrm{THz}$, the grating geometry was designed in a 70-nm (350-nm) length for a G1 (G2) finger with a 100-nm space. When an appropriate 2D electronic charge $\left(\sim 10^{12} \mathrm{~cm}^{-2}\right)$ is induced in the plasmon cavities under G1 while the regions under G2 are depleted, a strong electric field $(1 \sim 10 \mathrm{kV} / \mathrm{cm})$ arises at the plasmon cavity boundaries. When the device is photoexcited by laser irradiation, photoelectrons are dominantly generated in the regions under G2 and then are injected to the plasmon cavities under G1. If a specific drain-to-source bias is applied to promote a uniform slope along the source-to-drain direction on the energy band in the regions under G2, photoelectrons under G2 are unidirectionally injected to one side of the adjacent plasmon cavity. This may extensively accelerate the plasmon instability, leading to terahertz oscillation even at room temperature. The grating gates also act as terahertz antenna that converts non-radiative plasmon modes to radiative electromagnetic modes. Furthermore, a vertical cavity is formed in between the 2D-plasmon plane and the ITO mirror at the backside, which can effectively enhance the conversion gain and radiation power [4].

Photoresponse of the fabricated device to (a) a single CW laser and (b) 4-THz photomixed dual laser irradiation was first measured at room temperature. 1550-nm band tunable lasers were used. The fundamental resonant peaks of multi-mode self oscillations are observed in case of (a) (see Fig. 2(a)) under a certain $V_{\mathrm{G} 1}-V_{\mathrm{G} 2}$ condition so as to maximize the plasmon excitation efficiency. On the other hand, in Fig. 2(b), a resonant peak of injection-locked 4-THz oscillation is clearly observed, expecting successful photomixing operation. Significant improvement on the plasmon resonance (so far observed only at cryogenic temperature $[2,5]$ ) is owing to the original device structure.

Electromagnetic response to impulsive photoexcitation was measured at room temperature by using reflective electrooptic sampling (see Fig. 3) [6]. A 1.5- $\mu \mathrm{m}, 1$-mW, 70-fs laser pulse was used as pump and probe beams. The linearly polarized pump beam illuminated the device from the back surface. The probe beam, roughly cross-polarized to the pump beam, was led to the CdTe electrooptic sensor normal to the prism surface, fully reflecting at the CdTe surface back to the electrooptic detection block. The reflective index tensor is modulated by the local electric field so that the time-resolved electric field is detected as polarization change in the probe beam. Typical results and their Fourier spectra under appropriately biased and unbiased conditions are shown in Figs. 4-6. The temporal response for a biased sample without an ITO mirror exhibits a simple mono-pulse radiation, reflecting plasmon harmonic resonance (see Fig. 4). On the other hand, an appropriately biased sample with an ITO mirror emitted an impulsive radiation followed by monochromatic relaxation oscillation which was significantly enhanced by its vertical cavity (see Fig. 5). The Fourier spectrum exhibited resonant peaks at $0.8 \mathrm{THz}$ and its harmonic frequencies of up to 3.2 $\mathrm{THz}$ (see Fig. 6). These results are attributed to the emission of electromagnetic radiation stimulated by photo-induced plasmon instability. Estimated radiation power would exceed $10 \mu \mathrm{W}$.

The authors are grateful to Prof. T. Asano for his contribution and Dr. T. Nagatsuma for his supporting EOS measurement. This work was financially supported in part by the SCOPE program of MIC, Japan.

[1] M. Dyakonov, M. Shur, Phys. Rev. Lett., 71, 2465(1993). [4] T. Otsuji et al.,in IRMMW and THz Electron., 331(2004).

[2] S.A. Mikhailov, Phys. Rev. B, 58, 1517(1998).

[3] W. Knap et al., Appl. Phys. Lett., 84, 2331(2004).

[5] Y. Meziani et al., IEICE Trans. Electron., in press.

[6] L. Min, R.J.D. Miller, Appl. Phys. Lett., 56, 524(1990). 


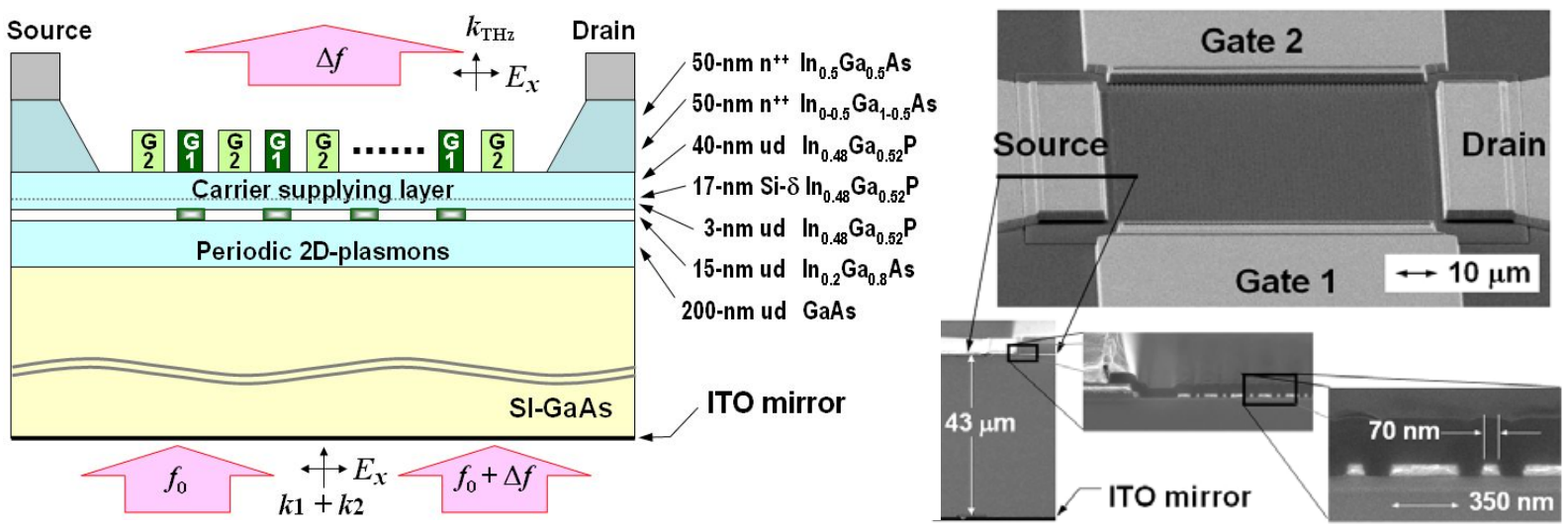

Fig. 1. Device structure and SEM images. $k_{1}, k_{2}$ : the wave vectors of irradiated photons, $E_{\chi}$ : the electric field (linear polarization), $k_{\mathrm{THz}}$ : the wave vector of electromagnetic radiation ( $\Delta f$ component).

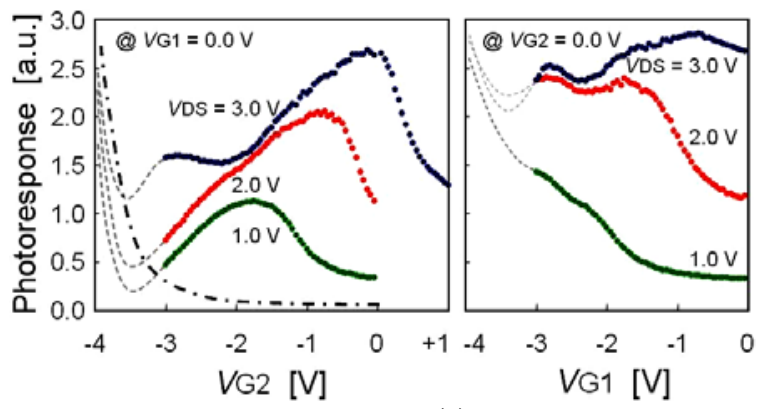

(a)

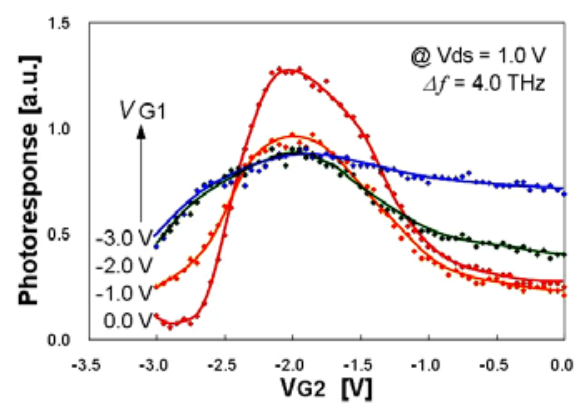

(b)

Fig. 2. Measured photoresponse showing excitation of plasmon instability. (a) a single CW laser irradiation, (b) 4-THz photomixed dual laser irradiation. Dotted line in (a) is for a standard HEMT, showing monotonic decrease with increase in gate bias.

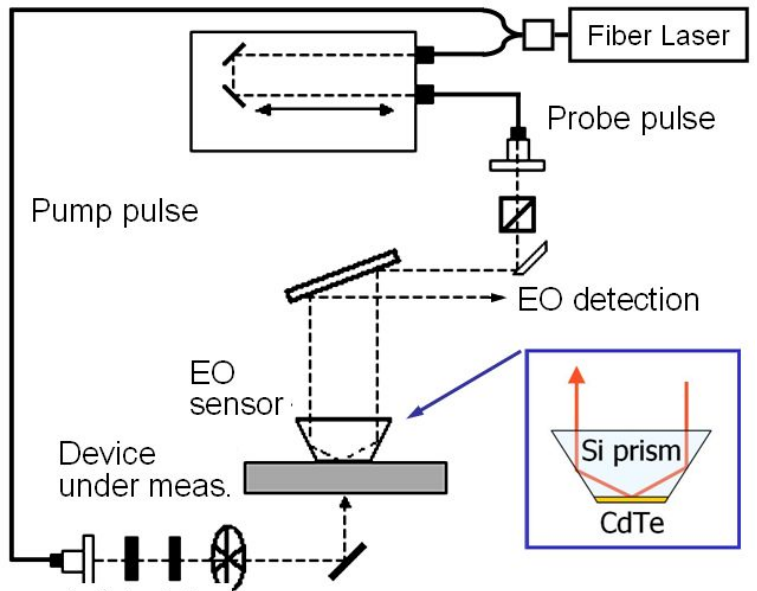

$\lambda / 2 \lambda / 4$

Fig. 3. Reflective electrooptic sampling system.

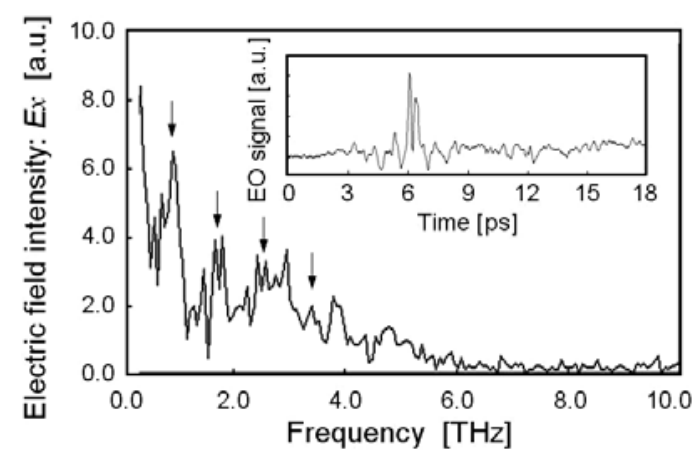

Fig. 4. Measured impulse response and its Fourier spectrum for a biased sample without ITO mirror.

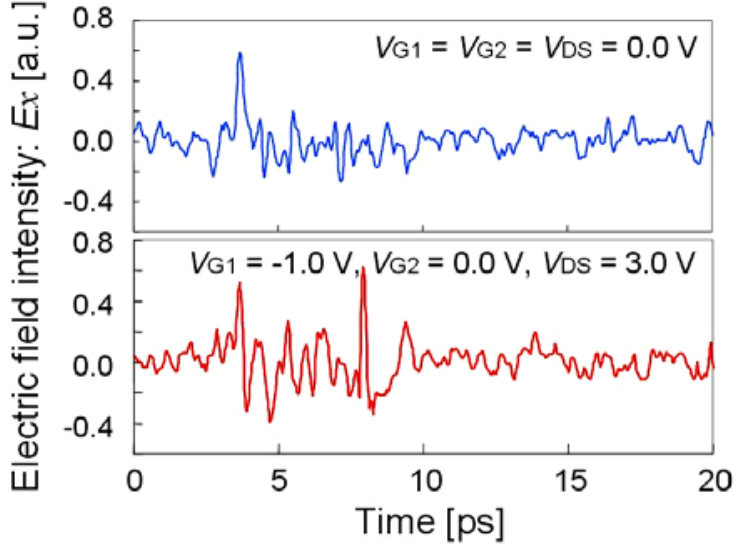

Fig. 5. Measured electromagnetic radiation to impulsive photoexcitation for a sample with ITO mirror. Upper: unbiased, lower: biased.

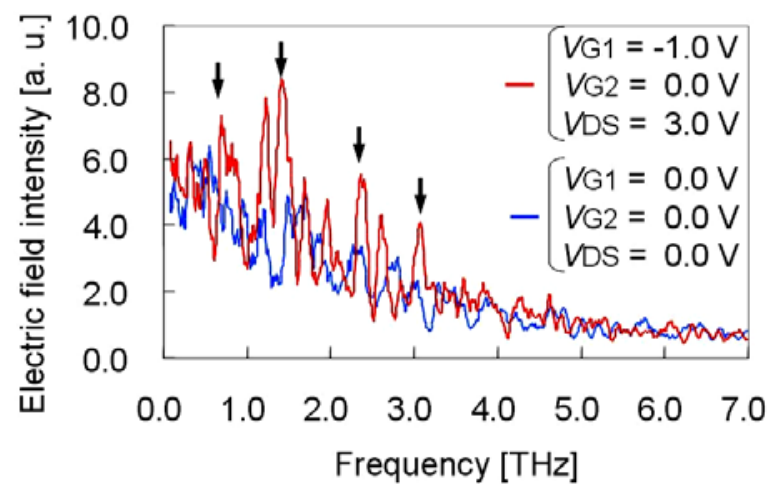

Fig. 6. Fourier spectrum for the results in Fig. 5. Red: biased, blue: unbiased. 